\title{
Mediodorsal thalamic lesions and object recognition in rats
}

\author{
DAVE G. MUMBY, JOHN P. J. PINEL, and FARHAD N. DASTUR \\ University of British Columbia, Vancouver, British Columbia, Canada
}

\begin{abstract}
Rats with bilateral electrolytic or sham lesions of the mediodorsal thalamic nucleus (MD) were tested on a nonrecurring-items delayed nonmatching-to-sample (DNMS) task-a test of object recognition. In Experiment 1, naive rats with MD lesions required more trials to solve the DNMS task at a 4-sec retention delay than did sham-lesion controls, and after they had done so, their performance was worse than that of the controls at delays ranging from 30 to $300 \mathrm{sec}$. In Experiment 2, rats with MD lesions that had received extensive DNMS training prior to surgery required more trials to remaster the task than did sham-lesion controls, and subsequently, their performance was worse than that of the controls at delays ranging from 4 to 300 sec. These find. ings resemble those reported in monkeys and humans with MD lesions, and they suggest that DNMS deficits in rats with MD lesions may be a useful component of a rat model of diencephalic amnesia.
\end{abstract}

Damage to certain structures of the medial diencephalon produces amnesia in humans (see, e.g., GraffRadford, Tranel, Van Hoesen, \& Brandt, 1990; Victor, Adams, \& Collins, 1971). Similarly, lesions of the mediodorsal thalamic nucleus (MD) in monkeys have been shown to disrupt the performance of the nonrecurringitems delayed nonmatching-to-sample (DNMS) task (Aggleton \& Mishkin, 1983a, 1983b; Zola-Morgan \& Squire, 1985), a test of object recognition that requires the kinds of memory abilities that are typically impaired in human amnesics (Gaffan, 1974; Mishkin, 1978). In the DNMS task, subjects must distinguish between an object that they have recently seen (i.e., the sample) and a different object that they have not. Normal monkeys can make such discriminations after sample-test delays of up to several minutes, but monkeys with MD lesions have difficulty at delays as brief as $10 \mathrm{sec}$ (Aggleton \& Mishkin, 1983b). Patients with diencephalic amnesia display similar deficits on the DNMS task (Squire, Zola-Morgan, \& Chen, 1988) and on the related delayed matching-to-sample task (Aggleton, Nicol, Huston, \& Fairbairn, 1988).

There have been many reports of impaired performance on memory tests by rats with MD lesions (e.g., Delacour, 1971; Kolb, 1977; Kolb, Pittman, Sutherland, \& Whishaw, 1982; M'Harzi, Jarrard, Willig, Palacios, \& Delacour, 1991; Stokes \& Best, 1990a, 1990b; Tigner, 1974; Winocur, 1985). However, because the tasks used in those studies bear little resemblance to the monkey DNMS task, the extent to which they involve similar

This research was supported by a grant to J.P.J.P. from the British Columbia Health Care Research Foundation. D.G.M. was supported by a postgraduate studentship from the Medical Research Council of Canada. We thank Robert Sutherland for his helpful comments on this manuscript. Correspondence should be addressed to D. G. Mumby, Department of Psychology, Logan Hall, University of New Mexico, Albuquerque, NM 87131-1161 (e-mail: mumby@bootes.unm.edu). memory abilities is unclear. In general, rat memory tasks typically require rats to remember places, whereas the monkey DNMS task requires monkeys to remember objects. Similar memory tasks for rats and monkeys would facilitate direct comparison of the amnesic effects of brain lesions in these two species.

In the present experiments, we assessed the effects of bilateral MD lesions on the ability of rats to learn and perform a DNMS task, in which, as in the monkey DNMS task, objects are used as test stimuli (Mumby, Pinel, \& Wood, 1990). Our primary objective in the present experiments was to determine whether the effects of MD lesions on object recognition in rats are comparable to those in primates. Severe DNMS deficits following MD lesions have been reported in monkeys that received all of their DNMS training after surgery (Zola-Morgan \& Squire, 1985) and in monkeys that were trained on the DNMS task prior to surgery (Aggleton \& Mishkin, 1983a, 1983b). Accordingly, we investigated the effects of MD lesions on DNMS performance in rats with no presurgery DNMS training (Experiment 1 ) and in rats with extensive presurgery DNMS training (Experiment 2).

\section{EXPERIMENT 1}

The ability of rats with bilateral MD lesions to solve the DNMS task at brief retention delays and then to perform it at longer delays was compared with that of shamlesion control rats. MD lesions produced deficits in both the acquisition and performance of DNMS over a wide range of retention delays.

\section{Method}

\section{Subjects}

The subjects were 8 experimentally naive, male Long-Evans rats (Charles River, Quebec) that were between 8 and 10 weeks old at 
the beginning of the experiment. They were housed individually with continuous access to water under a 12:12-h light:dark cycle, with light onset at 8:00 a.m. They had continuous access to food in their home cages until 7 days after surgery, at which time a restricted feeding regimen was introduced. Their body weights were reduced to approximately $85 \%$ of presurgery levels by giving them daily rations of rat chow; throughout the remainder of the experiment, they received approximately $25 \mathrm{~g}$ of rat chow per day. Training began after the rats had been on the restricted feeding regimen for 14 days (i.e., 21 days after surgery).

\section{Surgery}

Each rat received either bilateral electrolytic lesions $(n=5)$ or sham lesions $(n=3)$ of the MD. Surgery was conducted under pentobarbitol anesthesia. The lesions were made with a stainless steel bipolar electrode, which was insulated with Teflon except for approximately $1 \mathrm{~mm}$ at its tip. With the incisor bar positioned $3.3 \mathrm{~mm}$ below the interaural line, the electrode was lowered into the MD of each hemisphere, at the following coordinates, relative to bregma: AP $-2.8, \mathrm{ML}+0.6, \mathrm{DV}-6.5$. To produce the experimental lesions, $2 \mathrm{~mA}$ of current were passed through the electrode for $20 \mathrm{sec}$; to produce the sham lesions, the electrode was left in position for approximately $30 \mathrm{sec}$, but no current was passed through it.

\begin{abstract}
Apparatus
The apparatus has been described in detail elsewhere (Mumby et al., 1990). Briefly, it consists of an elevated runway, which is separated from identical goal areas at each end by opaque guillotine doors. Each goal area contains two food wells into which food pellets (45 mg, Bio-Serv Inc., Frenchtown, NJ) can be delivered by hand through plastic tubes that are mounted on the outside of the apparatus. A short divider wall, which protrudes from the center of the end wall, separates the two food wells. The sides of the goal areas are open to allow the experimenter to easily place stimulus objects over the food wells and to quickly remove them.

A collection of over 350 objects of various shapes, sizes, textures, and colors served as test stimuli. Each object was large enough to cover a food well but small enough and light enough to be easily displaced by the rats. No objects with obvious scents were included. The objects were washed, every few sessions, in a solution of water and chlorine bleach.
\end{abstract}

\section{Procedure}

All testing occurred during the light phase of the light:dark cycle, between 14 and $21 \mathrm{~h}$ after the rat's most recent meal. Each rat was tested no more than once per day and no fewer than four times per week. The rats were not handled during a session once they had been placed in the apparatus. Each rat was habituated to the apparatus (see Mumby et al., 1990), and then it progressed through three phases of training and testing: (1) acquisition of a simple two-choice object-discrimination task, (2) acquisition of the DNMS task, and (3) testing of DNMS performance at delays of $15,30,60,120$, and $300 \mathrm{sec}$. These procedures have been previously described in detail (see Mumby et al., 1990).

Object discrimination. For all of the subjects, the same two test objects served as the stimuli for the object-discrimination task. One of the objects was designated S+ (reward); the other object was designated $\mathrm{S}-$ (no reward). At the beginning of each trial, one of the guillotine doors was closed, and the experimenter positioned the $S+$ and $S-$ over the food wells on the other side of the door from the rat. Then, the experimenter opened the door, and the rat approached and displaced one of the objects. If the rat displaced $\mathrm{S}+$, a food pellet was delivered to that food well; if the rat displaced $\mathrm{S}-$, no food pellet was delivered. A rat was considered to have displaced an object only if it moved the object enough to ex- pose the food well. The experimenter then closed the far door and positioned the $S+$ and $S-$ over the food wells on the other side of it, in preparation for the next trial. The duration of the intertrial interval was typically between 15 and $20 \mathrm{sec}$. Rats were allowed to correct their errors on the first object-discrimination session-if the rat displaced $\mathbf{S}-$, it was allowed to then displace $\mathbf{S}+$ to obtain a reward before the experimenter removed the objects. Each objectdiscrimination session included 25 trials. Object-discrimination training continued for each rat until it achieved the criterion of at least 21 correct trials on a single session.

Acquisition of DNMS. The 350 test objects were divided into seven sets of 50 . Different sets were used on successive sessions, and different pairs of objects were used for each of the trials within a session. To begin each session, the rat was placed in the apparatus and permitted to explore it for approximately $1 \mathrm{~min}$. Then, the doors were lowered to enclose the rat in the central starting area. Before each trial, a food pellet was placed in one of the food wells, the sample object was placed over it, and the novel object was placed over one of the food wells at the other end of the apparatus.

To begin a trial, the experimenter raised the door to allow access to the sample object, which the rat approached and displaced from the food well. While the rat ate the pellet, the experimenter removed the sample object and positioned it over the vacant food well at the other end of the apparatus, behind the closed door. After the prescribed retention delay, the experimenter raised the door, and the rat approached and displaced either the sample object or the novel object. If it displaced the novel object, a food pellet was delivered to the exposed food well; if it displaced the sample object, no pellet was delivered. Then, the rat returned to the central starting area, and the experimenter closed the doors to confine it there until the next trial, which began after new sample and novel objects were positioned. The intertrial interval was typically between 20 and $30 \mathrm{sec}$. There were 20 DNMS trials per session.

The retention delay - the time between the displacement of the sample object by the rat and the raising of the door to reveal the sample and novel objects-was approximately 4 sec during DNMS acquisition training. For each rat, training continued at this 4-sec delay until it reached a criterion of at least 17 correct trials out of 20 on 2 consecutive sessions, or until it completed 75 sessions (i.e., 1,500 trials) without attaining this criterion. The rats were permitted to make corrections during the first two DNMS sessions, but not thereafter.

DNMS testing at longer delays. Only rats that attained the criterion within 75 sessions at the 4-sec delay were tested at longer delays. During this phase of testing, the delay was increased progressively to $15,30,60,120$, and $300 \mathrm{sec}$. Each rat received 8 sessions (i.e., 160 trials) at each delay.

\section{Behavioral}

\section{Results}

Table 1 summarizes the behavioral data for each of the rats in Experiment 1. The rats with MD lesions were impaired both in the rate at which they acquired the DNMS task at the 4-sec delay and in their subsequent DNMS performance at longer delays.

Object discrimination. All of the rats quickly solved the object-discrimination task. Table 1 shows the number of trials and the number of errors that each rat required to reach the criterion of 21 or more correct trials out of 25 on a single session; the trials and errors of the criterion session were excluded from the calculation of these measures. The rats with MD lesions averaged 70.0 trials and 21.0 errors to achieve criterion, whereas the 
Table 1

Acquisition of Object Discrimination and Delayed Nonmatchingto-Sample (Trials and Errors to Criterion) and Performance of Delayed Nonmatching-to-Sample (Percent Correct) by Rats With Mediodorsal Thalamic Lesions or Sham Lesions

\begin{tabular}{|c|c|c|c|c|c|c|c|c|c|}
\hline \multirow[b]{3}{*}{ Rat } & \multirow{2}{*}{\multicolumn{2}{|c|}{$\begin{array}{c}\text { Object } \\
\text { Discrimination }\end{array}$}} & \multicolumn{7}{|c|}{ Delayed Nonmatching-to-Sample } \\
\hline & & & \multicolumn{2}{|c|}{$4 \sec$} & \multirow[b]{2}{*}{$15 \mathrm{sec}$} & \multirow[b]{2}{*}{$30 \mathrm{sec}$} & \multirow[b]{2}{*}{$60 \mathrm{sec}$} & \multirow[b]{2}{*}{$120 \mathrm{sec}$} & \multirow[b]{2}{*}{$300 \mathrm{sec}$} \\
\hline & Trials & Errors & Trials & Errors & & & & & \\
\hline MDl & 100 & 39 & 1,420 & 506 & 75.0 & 72.5 & 64.0 & 56.5 & 62.5 \\
\hline MD2 & 75 & 19 & 880 & 342 & 76.5 & 65.5 & 59.5 & 58.0 & 51.5 \\
\hline MD3 & 25 & 8 & 960 & 314 & 73.0 & 78.0 & 66.5 & 67.0 & 58.0 \\
\hline MD4 & 100 & 24 & $1,500+$ & $688+$ & - & - & - & - & - \\
\hline MD5 & 50 & 15 & $1,500+$ & $650+$ & - & - & - & - & - \\
\hline$M$ & 70.0 & 21.0 & $1,252.0+$ & $500.0+$ & 74.8 & 72.0 & 63.3 & 60.5 & 57.3 \\
\hline SHI & 75 & 31 & 480 & 161 & 77.0 & 75.0 & 73.0 & 67.5 & 60.0 \\
\hline $\mathrm{SH} 2$ & 25 & 6 & 360 & 132 & 80.0 & 87.5 & 69.5 & 75.5 & 74.5 \\
\hline SH3 & 25 & 11 & 180 & 78 & 67.0 & 74.0 & 74.0 & 70.5 & 68.0 \\
\hline$M$ & 41.7 & 16.0 & 340.0 & 123.7 & 74.7 & 78.8 & 72.2 & 71.2 & 67.5 \\
\hline
\end{tabular}

Note-MD rats received bilateral lesions of the mediodorsal thalamus; $\mathrm{SH}$ rats received sham lesions. MD4 and MD5 were unable to reach the DNMS criterion at 4-sec delays within the maximum of 1,500 trials; thus, they were not tested at longer delays

rats with sham lesions averaged 41.7 trials and $16.0 \mathrm{er}-$ rors. Neither of these differences between the groups was statistically significant [trials, $t(6)=1.24, p=.263$; errors, $t(6)=.562, p=.599]$.

Acquisition of DNMS. The rats with MD lesions required significantly more trials to achieve the DNMS criterion than did the rats with sham lesions; the trials of the two criterion sessions were excluded from the calculation of this measure. In fact, 2 rats with MD lesions were unable to reach the criterion within the maximum of 1,500 trials; their mean scores over the last 200 trials were only slightly above chance levels $(M \mathrm{~s}=56.5 \%$ and $58.5 \%$ ), and thus they were not tested at longer delays. There was no overlap between the groups in either the number of trials or the number of errors that rats required to achieve the criterion $\left(U=0, n_{1} / n_{2}=3 / 5, p<.05\right)$.

DNMS testing at longer delays. The rats with MD lesions that managed to achieve the DNMS criterion performed more poorly than those with sham lesions at delays longer than $15 \mathrm{sec}$. There was no overlap between the scores in the two groups at delays of 60 and $120 \sec (U=$ $0, n_{1} / n_{2}=3 / 5, p<.05$ ).

\section{Histological}

Figure 1 illustrates the location and the extent of the MD lesions in Experiment 1. It is apparent in Figure 1 that there was near total bilateral destruction of the MD in all $5 \mathrm{MD}-\mathrm{lesion}$ rats and that nuclei adjacent to the MD were also damaged. There tended to be incomplete bilateral damage to the paraventricular, paratenial, paracentral, central medial, and habenular nuclei, as well as to the stria medullaris. There also tended to be small unilateral lesions in the anterodorsal, anteromedial, and anteroventral thalamic nuclei. Two of the MD-lesion rats had minor damage to the dentate gyrus, which was unilateral in one of them (MD3) and bilateral in the other
(MD4); their DNMS deficits were not worse than those of the MD-lesion rats in which the dentate gyrus was spared. The lesion in the rat with bilateral dentate gyrus damage is shown in Figure 1. We had previously observed normal DNMS performance at delays of up to $120 \mathrm{sec}$ in rats with large bilateral lesions of the hippocampal formation, which included the dentate gyrus (Mumby, Wood, \& Pinel, 1992).

\section{Discussion}

Bilateral lesions of the MD impaired the ability of rats to perform a DNMS task that closely resembles the DNMS task that is used to test object recognition in monkeys. Only 3 of the 5 rats with MD lesions were able to solve the task within the 1,500-trial limit, and these 3 rats required many more trials and errors to achieve the criterion than did any of the 3 control rats (see Table 1 ). The 3 rats with MD lesions that managed to achieve the criterion subsequently performed as well as controls at delays of $15 \mathrm{sec}$, but there was little or no overlap between the groups at delays of $\mathbf{3 0} \mathrm{sec}$ or longer.

The fact that all 5 of the rats with MD lesions readily solved the object-discrimination task-they all achieved the criterion within 4 days-suggests that their deficits on the DNMS task were not attributable to an inability to detect differences between the test objects and to respond on the basis of them. Supporting this conclusion is the lack of a statistically significant difference between the experimental and control rats in the rate at which they solved the object-discrimination task or the number of errors they made while solving it.

Deficits in the DNMS performance of brain-damaged animals are typically attributed to deficits in their ability to remember the sample objects over the retention delays (see Squire, 1992). However, when they are not thoroughly trained on the task prior to surgery, their deficits 


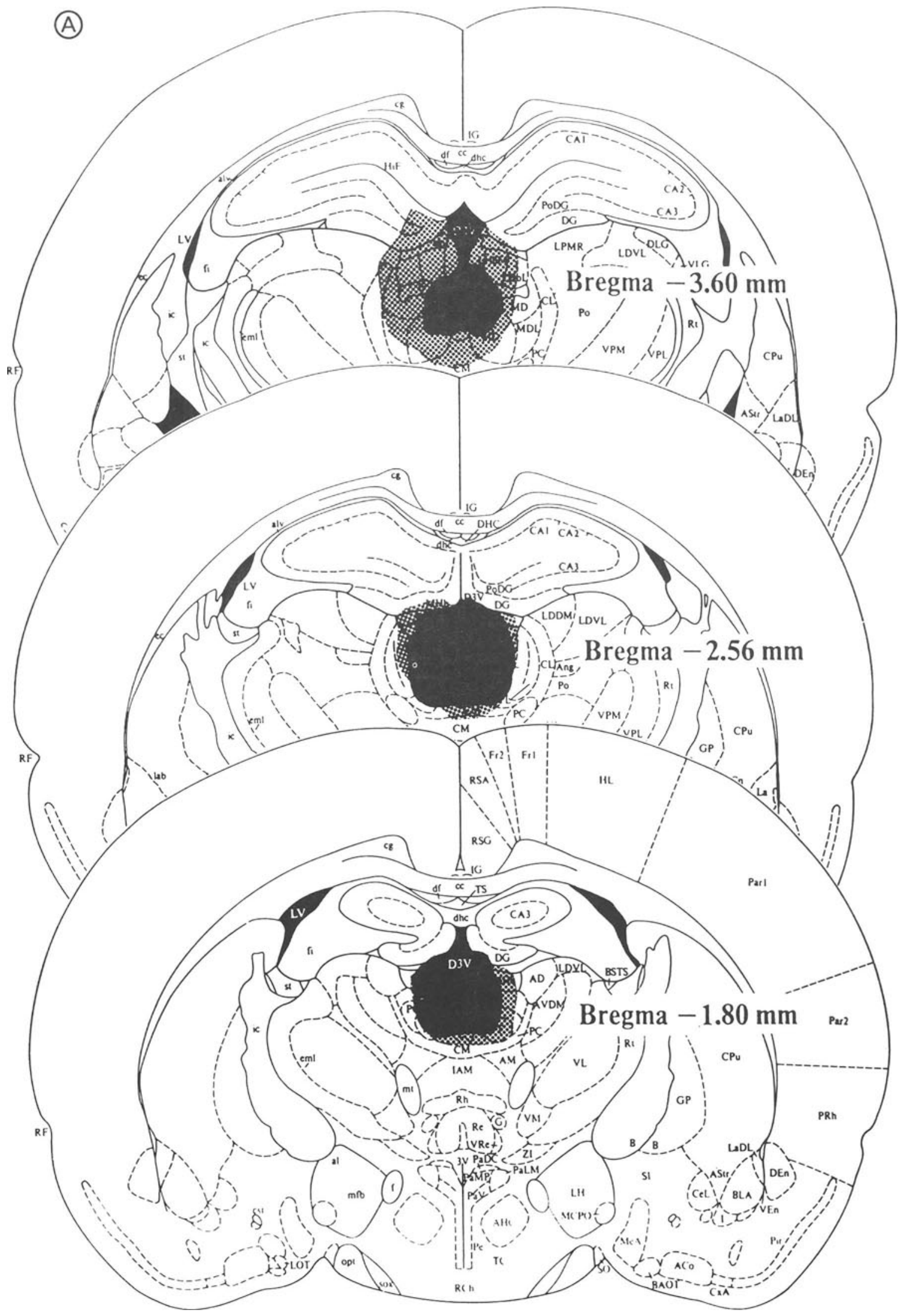


(B)

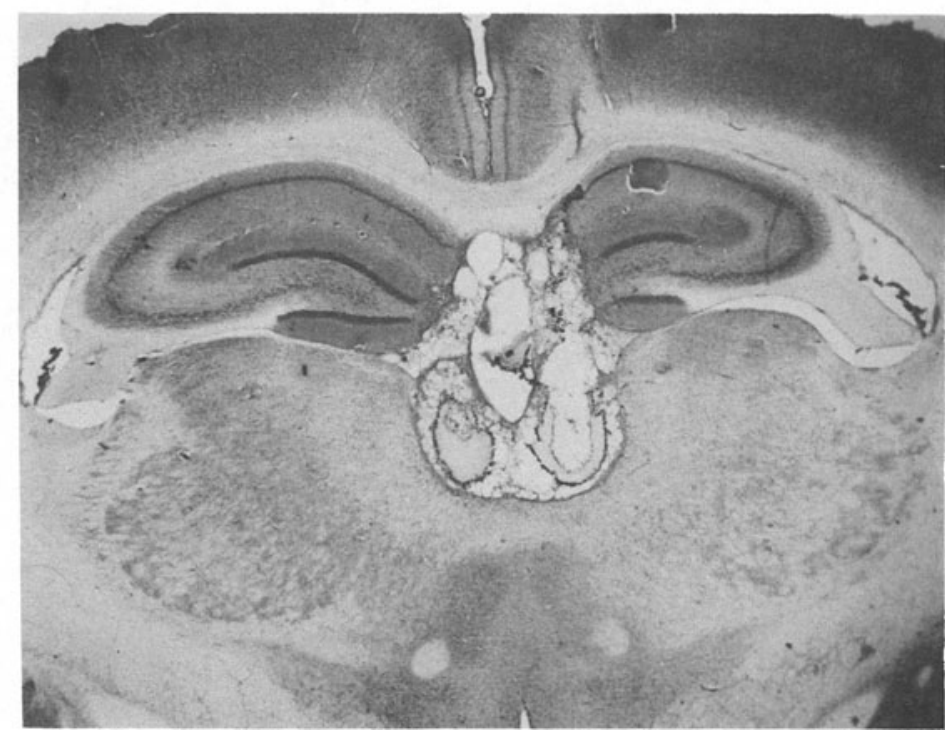

Figure 1. (A, facing page) Reconstructions of the smallest (black) and largest (gray) mediodorsal thalamic nucleus (MD) lesions in Experiment 1, based on the amount of thalamic damage. (B, above) The MD lesion from Experiment 1 that included bilateral damage to the dentate gyrus. One other rat sustained unilateral damage to the dentate gyrus. The severity of the DNMS deficit did not appear to be related to either the size of the MD lesion or the presence or absence of dentate-gyrus damage. The drawings were adapted from the atlas of Paxinos and Watson (1986).

are amenable to an alternative interpretation. It is possible that deficits in the DNMS performance of such subjects are a manifestation of learning deficits, rather than impaired object-recognition abilities. For example, in Experiment 1 , the 2 rats with MD lesions that could not achieve the criterion at the 4-sec delay may have been unable to solve the DNMS task because they could not acquire the nonmnemonic skills that are necessary to perform it, and the 3 rats with MD lesions that did eventually achieve the criterion at the 4-sec delay and that were then tested at longer delays may have had difficulty acquiring specific nonmnemonic skills (e.g., the ability to avoid distraction) that are necessary to reliably perform the task at longer delays (see Mumby et al., 1992). Two lines of evidence suggest that such learning deficits, in the absence of impaired object recognition, can contribute to the DNMS deficits that are observed in braindamaged animals: (1) the observation that DNMS deficits following medial-temporal-lobe lesions tend to be greater in animals that have not received training prior to surgery than in those that have (see Murray, 1990), and (2) the observation that DNMS performance at long delays of animals that have achieved stringent DNMS criteria at short delays gradually improves with practice (Mumby et al., 1990).

\section{EXPERIMENT 2}

The DNMS deficits of brain-damaged animals are generally attributed to amnesia, but there is another pos- sibility when the animals have not received DNMS training prior to surgery with the same range of retention delays at which they are later tested after surgery. In such instances, the DNMS deficits may be totally or partially attributable to impaired acquisition of procedural aspects of the task. The purpose of Experiment 2 was to confirm that MD lesions produce an anterograde object-recognition impairment in rats by ruling out the possible contribution of procedural-learning deficits. In Experiment 2, MD lesions produced DNMS deficits in rats that had received extensive training prior to surgery at all of the retention delays at which they were tested after surgery.

\section{Method}

\section{Subjects}

The subjects were 10 experimentally naive, male Long-Evans rats (Charles River, Quebec) that were between 8 and 12 weeks old at the beginning of the experiment. Their housing and daily feeding conditions were identical to those of the rats in Experiment 1. Training began after they had been on the restricted feeding regimen for 14 days.

\section{Procedure}

All testing occurred during the light phase of the light:dark cycle, between 14 and $21 \mathrm{~h}$ after the rat's most recent meal. Each rat was tested no more than once per day and no less than four times per week. Each rat was habituated to the apparatus, and then it progressed through four phases of training and testing: (1) acquisition of an object-discrimination task, (2) acquisition of the DNMS task at a 4-sec delay, (3) DNMS training with longer retention delays, and (4) determination of its presurgery DNMS retention function. Following recovery from surgery, each rat received two phases 
of testing: (1) reacquisition of the DNMS task at the 4-sec delay, and (2) determination of its postsurgery DNMS retention function.

Object discrimination and acquisition of DNMS. The general procedures for these two phases of training were identical to those of Experiment 1. Each rat was trained on a simple two-choice objectdiscrimination task until it reached the criterion of at least 21 correct trials out of 25 on a single session. Then it was trained on the DNMS task at delays of $4 \mathrm{sec}$ until it reached the criterion of at least 17 correct trials out of 20 on two consecutive sessions.

DNMS training at longer delays. Once a rat reached the criterion at delays of $4 \mathrm{sec}$, the delay was increased progressively to $15,30,60,120$, and $300 \mathrm{sec}$. Training at each delay continued until the rat either reattained the criterion of at least 17 correct trials out of 20 on two consecutive sessions, or until it completed 8 sessions (i.e., 160 trials) at that delay; a maximum of 4 sessions was administered at the 300 -sec delay.

Presurgery retention functions. Each rat's presurgery retention function was assessed during the final phase of testing prior to surgery. The rats received either 5 or 10 mixed-delay sessions, each consisting of 25 trials. On these sessions, 5 trials were conducted at each of the following delays: $4,15,60,120$, and $300 \mathrm{sec}$. These delays appeared in the following order in each session: $4,15,60$, $120,300,300,120,60,15,4,4,15 \mathrm{sec}$, and so on. Although we had previously found that performance was stable over 5 mixeddelay sessions (see Mumby et al., 1990), we thought that it might improve if the rats received more testing. Accordingly, some rats in the present experiment were given 10 mixed-delay sessions.

Surgery. Next, each rat received either bilateral MD lesions $(n=7)$ or sham lesions $(n=3)$. The surgical procedures were the same as those in Experiment 1 . The rats were allowed 14 days to recover from surgery before testing resumed. During the first 10 days after surgery, the rats had continuous access to food, after which they were returned to the restricted feeding regimen.

Postsurgery reacquisition of DNMS. Following recovery, the rats were tested on the DNMS task at 4-sec delays until they either reattained the original performance criterion or completed 20 sessions without reattaining it.

Postsurgery retention functions. Next, each rat's postsurgery retention function was determined by using the same procedures that had been used to determine its presurgery retention function. Each rat received the same number of mixed-delay sessions after surgery as it had received before surgery (i.e., either 5 or 10).

Results
Behavioral
Relative to the rats with sham lesions, the rats with MD lesions were severely impaired following surgery in terms of both the rate at which they remastered the DNMS task at the 4-sec delay and their DNMS performance at longer delays.

Object discrimination, acquisition of DNMS, and DNMS training at longer delays. The 10 rats required an average of $55.0(S E=7.27)$ trials and $18.4(S E=$ 2.56) errors to reach the criterion on the objectdiscrimination task, and $336.0(S E=60.95)$ trials and $120.2(S E=23.25)$ errors to reach criterion on the DNMS task; these means were comparable to the corresponding means for the sham-lesion controls in Experiment 1 (see Table 1). During DNMS training at progressively longer delays, scores decreased with each increment in the delay. The number of rats reattaining criterion within the maximum of eight sessions at delays of $15,30,60,120$, and $300 \mathrm{sec}$ was $8,9,3,3$, and 0 rats, respectively.
Presurgery retention functions. Figure 2 illustrates the presurgery retention functions for the rats that subsequently received MD lesions and for those that subsequently received sham lesions. Repeated measures analyses of variance (ANOVAs) indicated that the scores in each group declined significantly as the delay was lengthened [MD lesion, $F(4,20)=14.72, p<.001$; sham lesion, $F(4,8)=8.32, p<.01]$. Planned comparisons revealed no significant differences at any delay between the scores of the two groups prior to surgery (all $p s>.05$ ).

Reacquisition of DNMS following surgery. Each rat readily displaced test objects from the food wells during the first session following surgery and continued to do so thereafter. Figure 3 illustrates the number of trials that each rat required to reattain the DNMS criterion at the 4-sec delay after surgery; the trials of the two criterion sessions were excluded from the calculation of this measure. All three of the sham-lesion rats achieved the criterion on the first two sessions (i.e., they required 0 trials to reattain the criterion), whereas none of the $7 \mathrm{MD}$-lesion rats did-in fact, 3 of the MD-lesion rats failed to reattain the criterion within the maximum of 400 trials. Two of them scored $90 \%$ or better on more than one session, and they averaged $73 \%$ and $78 \%$, respectively, over their final 80 trials. The postsurgery retention functions for these 2 rats were determined and included in the following analyses, despite the fact that inconsistent scores from day to day had kept them from achieving the criterion. The other one never scored above $70 \%$ on any session following surgery, and it averaged only $55 \%$ correct over its final 80 trials. This rat received no further testing, and its data were excluded from the following comparisons between the MD-lesion rats' presurgery and postsurgery retention functions, as well as from this group's mean presurgery retention function shown in Figure 2.

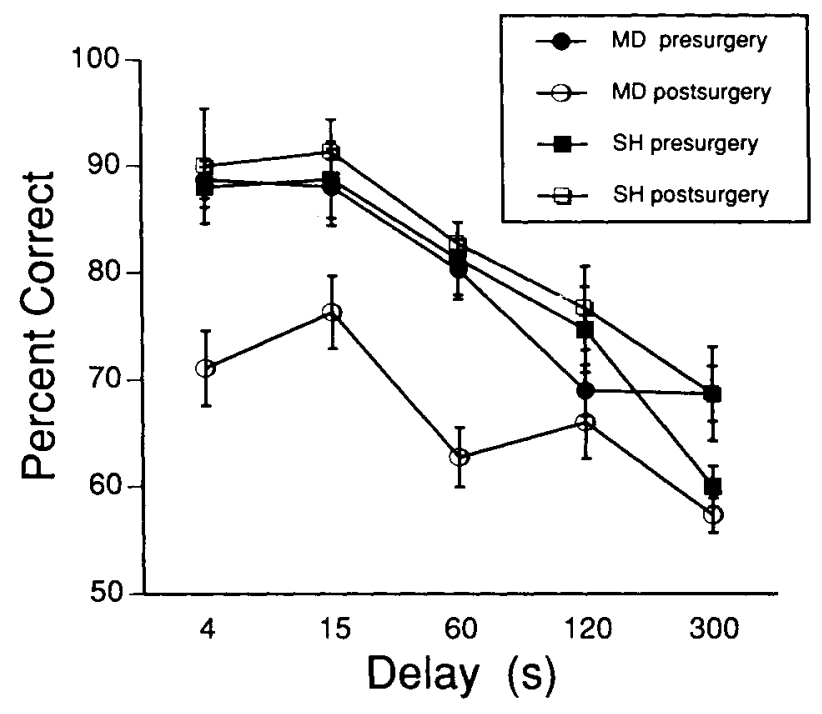

Figure 2. Mean presurgery and postsurgery retention functions for rats with mediodorsal thalamic nucleus (MD) lesions and rats with sham (SH) lesions. The error bars show SEMs. 


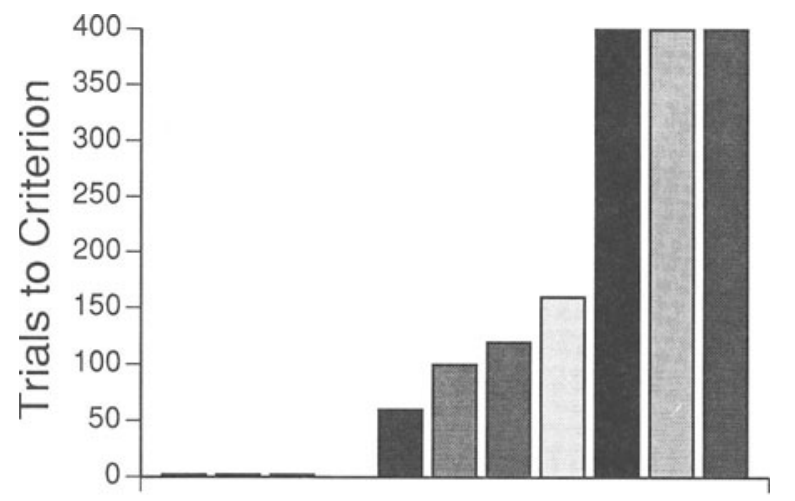

SH1 SH2 SH3 MD1 MD2 MD3 MD4 MD5 MD6 MD7

\section{Rat}

Figure 3. The number of postsurgery delayed nonmatching-tosample trials that individual rats required to reattain the criterion at the 4 sec delay. All 3 of the rats with sham (SH) lesions performed at criterion levels on the first two postsurgery sessions, whereas none of the rats with mediodorsal thalamic nucleus (MD) lesions did. Three of the MD-lesion rats were unable to reach the criterion within the maximum of $\mathbf{4 0 0}$ trials.

Postsurgery retention functions. Figure 2 illustrates the postsurgery retention functions for both groups. Planned comparisons of the number of correct trials at each delay indicated that, following surgery, the performance of rats with MD lesions was significantly worse than that of rats with sham lesions at all delays, with the exception of the 120 - sec delay $[4 \mathrm{sec}, t(7)=2.73, p<$ $.02 ; 15 \mathrm{sec}, t(7)=2.50, p<.02 ; 60 \mathrm{sec}, t(7)=4.18$, $p<.01 ; 120 \mathrm{sec}, t(7)=1.71, p<.10 ; 300 \mathrm{sec}, t(7)=$ $2.69, p<.02$; one-tailed tests]. Moreover, repeated measures ANOVAs indicated that the performance of the rats with MD lesions was significantly poorer after surgery than before $[F(1,5)=18.50, p<.01]$. In contrast, the scores for the sham-lesion rats following surgery were not significantly different from their scores before surgery at any delay. Repeated measures ANOVAs revealed that the scores of both groups following surgery declined significantly as the delay was lengthened [MD lesion, $F(4,20)=4.80, p<.001$; sham lesion, $F(4,8)=4.89$, $p<.05]$. A one-sample $t$ test revealed that the scores of the rats with MD lesions were still significantly better than chance at the 300-sec delay $[t(5)=4.71, p<.01$, one-tailed].

\section{Histological}

Figure 4 illustrates the location and extent of the MD lesions in Experiment 2. The extent of thalamic damage was similar to that in Experiment 1 (see Figure 1). In addition to their thalamic lesions, 1 of the MD-lesion rats in Experiment 2 sustained bilateral damage to the dentate gyrus and 2 of them sustained unilateral damage to the dentate gyrus.

\section{Discussion}

Bilateral lesions of the MD impaired the DNMS performance of rats that had received extensive DNMS training prior to surgery. Relative to rats with sham lesions. rats with MD lesions were impaired in terms of both the rate at which they remastered the DNMS task at the 4sec delay and the accuracy of their DNMS performance at delays ranging from 4 to $300 \mathrm{sec}$. The sham-lesion rats displayed complete savings of the DNMS abilities that they had acquired before surgery; they performed at asymptotic levels on the very first session following surgery, which was conducted at the 4-sec delay. In contrast, 3 of the $7 \mathrm{MD}$-lesion rats were unable to reattain the DNMS criterion at the 4-sec delay within the 20-session (i.e., 400-trial) limit, and the others required several sessions to achieve the same criterion ( $M=5.5$ sessions). Moreover, performance of the MD-lesion rats on mixeddelay sessions was significantly worse than both their own performance prior to surgery and the performance of the sham-lesion rats following surgery at delays ranging from 4 to $300 \mathrm{sec}$ (see Figure 2).

During reacquisition of the DNMS task, most of the MD-lesion rats eventually reached the criterion of $85 \%$ correct on two consecutive sessions at the 4-sec delay. However, when they were subsequently tested on mixeddelay sessions, their scores at the 4-sec delay were much lower than the 17 -out-of-20 (i.e., $85 \%$ ) criterion ( $M=$ $71 \%$; see Figure 2). The sham-lesion rats did not display a similar effect-their scores at the 4-sec delay on mixeddelay sessions averaged $90 \%$, which was similar to the average score on their final DNMS reacquisition sessions. The change in procedures, from sessions where the delay was the same on all trials (i.e., during reacquisition) to sessions where the delay changed from trial to trial (i.e, mixed-delay sessions), appeared to disrupt the ability of MD-lesion rats to perform the DNMS task at the 4-sec delay. A similar reduction in scores has been reported in amnesic patients when they were switched from sessions in which all trials were conducted at a 5-sec delay to sessions in which only some of the trials were conducted at a 5-sec delay and the other trials were conducted at longer delays (Squire et al., 1988). One possibile explanation for this effect is that the inevitable errors made by subjects with MD lesions at longer delays have a disruptive effect on their performance at brief delays during the same session. In any case, this interesting parallel between the DNMS performance of rats and humans with MD damage lends support to the idea that DNMS deficits in MD-lesion rats model features of human diencephalic amnesia.

The observation in Experiment 2 of severe DNMS deficits in MD-lesion rats that had received extensive training prior to surgery strongly suggests that MD lesions produce an impairment of object recognition. The DNMS deficits cannot be attributed to impaired acquisition of nonmnemonic skills that are required for performance of the DNMS task, because extensive training was conducted before surgery at all of the retention delays that 


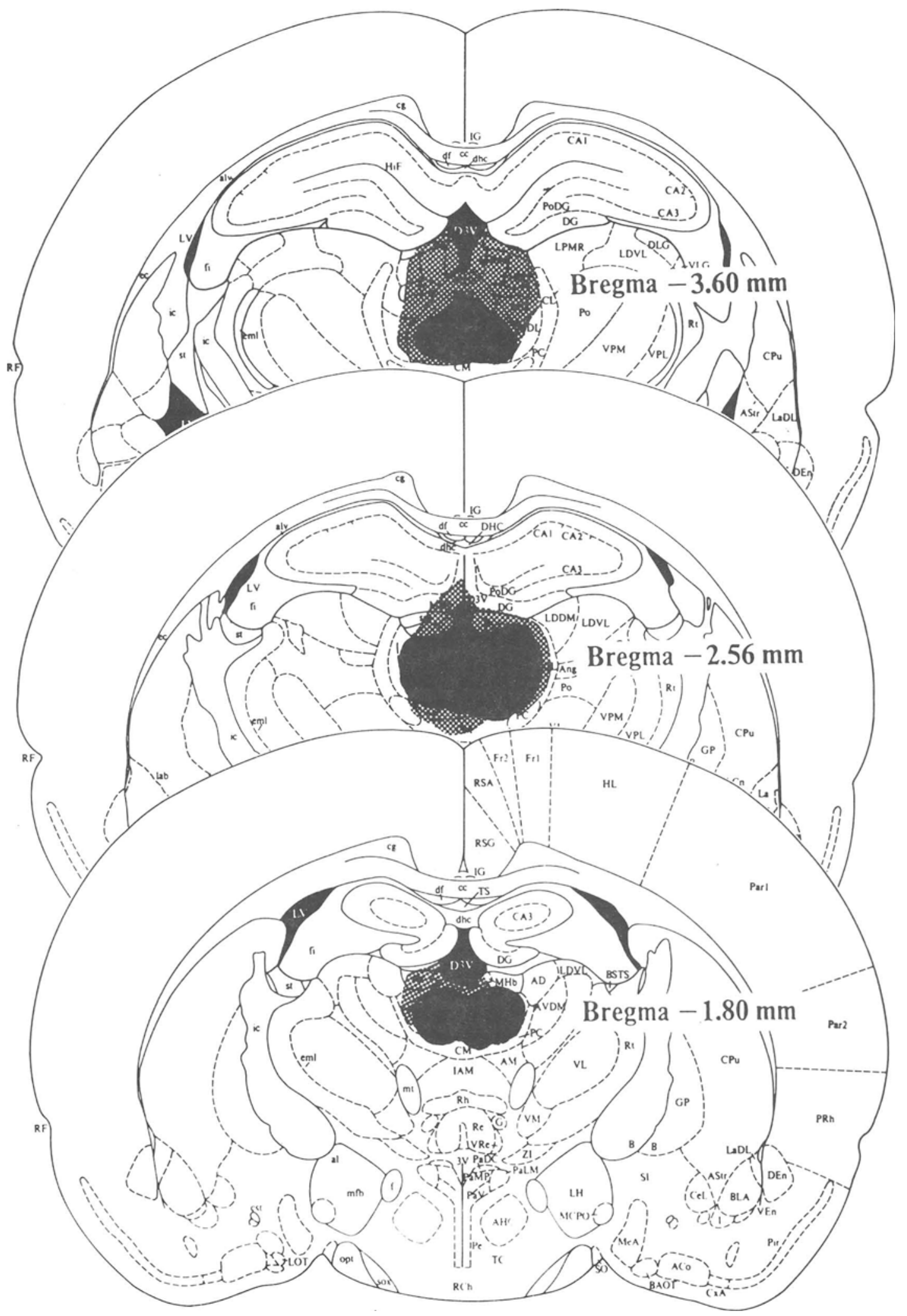

Figure 4. Reconstructions of the smallest (black) and largest (gray) mediodorsal thalamic nucleus (MD) lesions in Experiment 2, based on the amount of thalamic damage. 
were employed following surgery. It is possible that the MD lesions had retrograde effects on the retention of these skills; however, the observation that all 7 of the MD-lesion rats readily approached and displaced test objects on the first DNMS session following surgery indicates that they remembered the major procedural aspects of the task.

\section{GENERAL DISCUSSION}

Bilateral lesions of the MD produced DNMS deficits both in rats that received no training prior to surgery (Experiment 1) and in rats that received extensive DNMS training prior to surgery (Experiment 2). These deficits were similar to those that have been reported in monkeys and humans with MD lesions in at least six ways: (1) The MD-lesion rats that were not trained prior to surgery required a greater number of trials to solve the DNMS task than did control rats; a similar observation has been reported in monkeys (Zola-Morgan \& Squire, 1985) and in amnesic humans (Squire et al., 1988). (2) After the MD-lesion rats that had not received training prior to surgery had mastered the DNMS task at a brief (i.e., 4-sec) delay, increasing the duration of the delay reinstated their DNMS deficits (see Table 1); a similar observation has been reported in monkeys with MD lesions (Zola-Morgan $\&$ Squire, 1985) and in amnesic humans (Squire et al., 1988). (3) The MD-lesion rats that received extensive DNMS training prior to surgery required more trials to remaster the task than did control rats; a similar observation has been reported in monkeys with MD lesions ( $\mathrm{Ag}$ gleton \& Mishkin, 1983a, 1983b). (4) After they had remastered the task at the 4-sec delay, the MD-lesion rats that had received extensive DNMS training prior to surgery performed worse than controls over a wide range of delays (i.e., 4 to $300 \mathrm{sec}$ ), not just at long delays; similar DNMS deficits have been reported in monkeys with MD lesions (Aggleton \& Mishkin, 1983a, 1983b). (5) The DNMS performance of MD-lesion rats at a brief (i.e., 4-sec) delay was worse when only some of the trials within a session had the brief delay than when all of the trials within a session had the brief delay; a similar observation has been reported in human amnesics (Squire et al., 1988). (6) Despite their DNMS deficits, MD-lesion rats easily solved a simple object-discrimination task; monkeys with MD lesions that are impaired on the DNMS task solve visual pattern discriminations at a normal rate (Aggleton \& Mishkin, 1983a; Zola-Morgan \& Squire, 1985).

Although DNMS deficits in brain-damaged subjects are commonly attributed to anterograde amnesia, the DNMS deficits that were displayed in Experiment 1 by MD-lesion rats may have in part reflected an impairment in their ability to learn or apply rules of performance (i.e., the nonmatching principle) or procedural skills (e.g., attending to the appropriate cues, avoiding distraction, suppressing response biases) because they were not trained on the DNMS task until after their surgery. In contrast, the MDlesion rats in Experiment 2 received DNMS training prior to surgery at the same delays that were employed follow- ing surgery. Their DNMS deficits did not result from an inability to acquire the necessary rules of performance and procedural skills because these had been acquired prior to surgery. It is likely that impaired recognition abilities contributed their DNMS deficits. Although it is possible that the MD lesions caused a retrograde impairment in the retention of procedural skills, the observation that all of the rats readily approached and displaced test objects on the first session following surgery suggests that they retained the major procedural aspects of the task.

The present results suggest that the rat DNMS task may be useful in modeling diencephalic amnesia in rats. The advantage of the present DNMS paradigm is that it is directly comparable to the DNMS paradigm that is used to study object recognition in monkeys. The similarity of the rat and monkey DNMS tasks provides a broader comparative basis for making inferences about the anatomical bases of object recognition-one that includes rats as well as monkeys.

On the basis of findings in brain-damaged monkeys, Mishkin (1982) proposed a model of the neural system that underlies object recognition. The proposed model system comprises two subsystems-one that involves the amygdaloid complex and the MD, and one that involves the hippocampal formation and the anterior thalamic nuclear complex, which is situated adjacent to MD (Mishkin, 1982). Each of these subsystems can compensate for damage in the other subsystem; a severe impairment of object-recognition abilities will only result from damage that disrupts the functions of both subsystems. Support for this model comes from the observation that combined bilateral lesions of both the hippocampus and the amygdala, or their primary efferents, produce a severe DNMS impairment-one that is greater than would be expected from a simple summation of the relatively mild impairment produced by bilateral lesions to either structure, or their efferent systems, alone (Bachevalier, Parkinson, \& Mishkin, 1985; Mishkin, 1978; Murray \& Mishkin, 1984; Zola-Morgan \& Squire, 1986). When the results of the present experiments are considered together with those of a recent study on the effects of hippocampal and amygdalar lesions on DNMS in rats (Mumby et al., 1992), it does not appear that the dual system that was described by Mishkin underlies object recognition in rats. In that earlier study (Mumby et al., 1992), extensive bilateral lesions of both the hippocampus and the amygdala produced only mild DNMS deficits in rats with extensive presurgery DNMS training-those rats were unimpaired at retention delays of up to $120 \mathrm{sec}$, and they were impaired only at a delay of $600 \mathrm{sec}$ (cf. Aggleton, Blindt, \& Rawlins, 1989). By contrast, in the present study, bilateral lesions in the medial thalamus produced a profound DNMS deficit that was apparent at retention delays as brief as $4 \mathrm{sec}$.

Although the possibility cannot be ruled out that the DNMS deficits observed in the present study were the result of incidental damage to thalamic structures other than the MD, the present findings clearly suggest a role for 
medial thalamic structures in object recognition in rats. More studies are needed to determine the contribution that specific medial thalamic nuclei and fiber tracts passing through this region make to object recognition and other memory abilities. A variety of memory tasks will be required in order to fully characterize the nature of the memory deficits in brain-damaged rats. One useful approach to devising a battery of memory tasks for this purpose would be to follow the strategy that was employed in the development of the present rat DNMS task-that is, to design additional memory tasks for rats that mimic those that are used to study amnesia in monkeys. A collection of appropriately designed tasks would facilitate direct comparisons of brain-memory relations in rats and monkeys.

\section{REFERENCES}

Aggleton, J. P., Blindt, H. S., \& Rawlins, J. N. P. (1989). Effects of amygdaloid and amygdaloid-hippocampal lesions on object recognition and spatial working memory in rats. Behavioral Neuroscience, 103, 962-974

Aggleton, J. P., \& Mishkin, M. (1983a). Memory impairments following restricted medial thalamic lesions in monkeys. Experimental Brain Research, 52, 199-209.

Aggleton, J. P., \& Mishkin, M. (1983b). Visual recognition impairments following medial thalamic lesions in monkeys. Neuropsychologia, 21, 189-197.

Aggleton, J. P., Nicol, R. M., Huston, A. E., \& Fairbairn, A. F. (1988). The performance of amnesic subjects on tests of experimental amnesia in animals: Delayed matching-to-sample and concurrent learning. Neuropsychologia, 26, 265-272.

Bachevalier, J., Parkinson, J. K., \& Mishkin, M. (1985). Visual recognition in monkeys: Effects of separate vs. combined transection of fornix and amygdalofugal pathways. Experimental Brain Research, 57, 554-561.

Delacour, J. (1971). Effects of medial thalamic lesions in the rat: A review and an interpretation. Neuropsychologia, 9, 157-174.

GaFFAN, D. (1974). Recognition impaired and association intact in the memory of monkeys after transection of the fornix. Journal of Comparative \& Physiological Psychology, 86, 1100-1109.

Graff-Radford, N. R., Tranel, D., Van Hoesen, G. W., \& Brandt, J. P. (1990). Diencephalic amnesia. Brain, 113, 1-25.

KoLB, B. (1977). Studies on the caudate-putamen and the dorsomedial thalamic nucleus of the rat: Implications for mammalian frontal lobe functions. Physiology \& Behavior, 18, 237-244.

Kolb, B., Pittman, K., Sutherland, R. J., \& Whishaw, I. Q. (1982). Dissociations of the contribution of the prefrontal cortex and dorsomedial thalamic nucleus to spatially guided behavior in the rat. Behavioural Brain Research, 6, 365-378.
M'Harzi, M. Jarrard, L. E., Willig, F., Palacios, A., \& Delacour, J. (1991). Selective fimbria and thalamic lesions differentially impair forms of working memory. Behavioral \& Neural Biology, 56, 221-239.

Mishkin, M. (1978). Memory in monkeys severely impaired by combined but not by separate removal of amygdala and hippocampus. Nature, 273, 297-298.

Mishkin, M. (1982). A memory system in the monkey. Philosophical Transactions of the Royal Society of London, 298, 85-95.

Mumby, D. G., Pinel, J. P. J., \& WoOd, E. R. (1990). Nonrecurringitems delayed nonmatching-to-sample in rats: A new paradigm for testing nonspatial working memory. Psychobiology, 18, 321-326.

Mumby, D. G., Wood, E. R., \& Pinel, J. P. J. (1992). Objectrecognition memory is only mildly impaired in rats with lesions of the hippocampus and amygdala. Psychobiology, 20, 18-27.

MurRay, E. A. (1990). Representational memory in nonhuman primates. In R. P. Kesner \& D. S. Olton (Eds.), Neurobiology of comparative cognition (pp. 127-155). Hillsdale, NJ: Erlbaum.

MurRay, E. A., \& Mishin, M. (1984). Severe tactual as well as visual memory deficits follow combined removal of the amygdala and hippocampus in monkeys. Journal of Neuroscience, 4, 2565-2580.

PAXINOS, G., \& WATSON, C. (1986). The rat brain in stereotaxic coordinates (2nd ed.). Orlando, FL: Academic Press.

SQuire, L. R. (1992). Memory and the hippocampus: A synthesis from findings with rats, monkeys, and humans. Psychological Review, 99 , 195-231.

Squire, L. R., Zola-Morgan, S., \& Chen, K. S. (1988). Human amnesia and animal models of amnesia: Performance of amnesic patients on tests designed for the monkey. Behavioral Neuroscience, 102, 210-221.

StOKES, K. A., \& BEST, P. J. (1990a). Mediodorsal thalamic lesions impair "reference" and "working" memory in rats. Physiology \& Behavior, 47, 471-476.

Stokes, K. A., \&est, P. J. (1990b). Mediodorsal thalamus lesions in rats impair radial-arm maze performance in a cued environment. Psychobiology, 18, 63-67.

TIGNER, J. C. (1974). The effects of dorsomedial thalamic lesions on learning, reversal, and alternation behavior in the rat. Physiology \& Behavior, 12, 13-17.

Victor, M., Adams, R. D., \& Collins, G. H. (1971). The WernickeKorsakoff syndrome. Oxford: Blackwell.

WINOcuR, G. (1985). The hippocampus and thalamus: Their roles in short- and long-term memory and the effects of interference. Behavioural Brain Research, 16, 135-152.

Zola-Morgan, S., \& SQuire, L. R. (1985). Amnesia in monkeys following lesions of the mediodorsal nucleus of the thalamus. Annals of Neurology, 17, 558-564.

Zola-Morgan, S., \& SQuire, L. R. (1986). Memory impairment in monkeys following lesions of the hippocampus. Behavioral Neuro science, 100, 165-170.

(Manuscript received August 28, 1992; revision accepted for publication November $12,1992$. ) 